\title{
Dark-matter spike at the galactic center?
}

\author{
Piero Ullio* \\ California Institute of Technology, Mail Code 130-33, Pasadena, California 91125 \\ and SISSA, via Beirut 4, 34014 Trieste, Italy \\ HongSheng Zhao ${ }^{\dagger}$ \\ Institute of Astronomy, Madingley Road, Cambridge, CB3OHA, United Kingdom \\ Marc Kamionkowski ${ }^{*}$ \\ California Institute of Technology, Mail Code 130-33, Pasadena, California 91125
}

(Received 29 January 2001; published 20 July 2001)

\begin{abstract}
The past growth of the central black hole (BH) might have enhanced the density of cold dark matter halo particles at the galactic center. We compute this effect in realistic growth models of the present $(2-3)$ $\times 10^{6} M_{\odot} \mathrm{BH}$ from a low-mass seed $\mathrm{BH}$, with special attention to dynamical modeling in a realistic galaxy environment with merger and orbital decay of a seed BH formed generally outside the exact center of the halo. An intriguing "very-dense spike" of dark matter has been claimed in models of Gondolo and Silk with a density high enough to contradict with experimental upper bounds of neutralino annihilation radiation. This "spike" disappears completely or is greatly weakened when we include important dynamical processes neglected in their idealized or restrictive picture with cold particles surrounding an at-the-center zero-seed adiabatically growing $\mathrm{BH}$. For the seed $\mathrm{BH}$ to spiral in and settle to the center within a Hubble time by dynamical friction, the seed mass must be at least a significant fraction of the present $\mathrm{BH}$. Any subsequent at-the-center growth of the $\mathrm{BH}$ and steepening of the central Keplerian potential well can squeeze the halo density distribution only mildly, whether the squeezing happens adiabatically or instantaneously.
\end{abstract}

DOI: 10.1103/PhysRevD.64.043504

PACS number(s): 95.35.+d, 14.80.Ly, 95.55.Vj

\section{INTRODUCTION}

Weakly interacting massive particles (WIMPs) are among the leading candidates for the dark matter in galactic halos. Such particles arise in extensions to the standard model of particle physics. The most widely studied example is the neutralino, plausibly the lightest superpartner in supersymmetric versions of the standard model. The cosmological abundance of WIMPs is naturally of the order of the critical density of the Universe, and so WIMPs make natural darkmatter candidates.

The search for WIMPs in the Milky Way halo has been a major endeavor both theoretically and experimentally in the last two decades (for comprehensive reviews see Refs. [1,2]). The main efforts so far have been to detect WIMPs directly in low-background laboratory experiments and indirectly via observation of the energetic neutrinos produced by annihilation of WIMPs that have accreted in the Sun and/or Earth. Another possibility is to search for exotic cosmic rays, such as high-energy gamma rays, positrons, antiprotons, or neutrinos produced by WIMP pair annihilations in the galactic halo. In particular, the flux of gamma rays in a given direction is proportional to the line-of-sight integral of the square of the WIMP density. Since the dark-matter density is expected to be largest towards the galactic center, the flux of such exotic gamma rays should be highest in that direction.

\footnotetext{
*Email address: ullio@he.sissa.it

†Email address: hsz@ast.cam.ac.uk

${ }^{\ddagger}$ Email address: kamion@ @apir.caltech.edu
}

We now know that black holes of mass of $10^{6}-10^{8} \mathrm{M}_{\odot}$ reside in most galactic centers (see, e.g., [3]). In particular, the arguments for a $2.6 \times 10^{6} \mathrm{M}_{\odot}$ black hole at the center of the Milky Way are at this point almost incontrovertible (see, e.g., Ref. [4]) given the rise of the velocity (radial and proper motion) dispersion within $1 \mathrm{pc}$ of the galactic center. It has long been argued that if a black hole grows inside a given population of stars, the density of stars should be enhanced in the vicinity of the black hole after its appearance [5-8]. If the WIMP density is likewise enhanced at the galactic center, then the source of exotic cosmic rays from WIMP annihilation therein should be increased. Several authors investigated the enhancement in the gamma-ray flux for the dark-matterdensity spike, $\rho(r) \propto r^{-1.5}$ as the radius $r \rightarrow 0$, that would be induced by the adiabatic growth of a black hole in an initially isothermal dark-matter profile [7].

More recently, Gondolo and Silk [9] (GS) investigated the dark-matter spike ${ }^{1}$ that arises if a black hole grows adiabatically at the center of a dark-matter halo that initially has a singular power-law cusp, $\rho(r) \propto r^{-\gamma}$ with $0<\gamma<2$, as suggested by numerical simulations $[10,11]$. They found that the black-hole growth gives rise to a dark-matter spike $\rho(r)$ $\propto r^{-A}$ with $2.25 \leqslant A \leqslant 2.5$, in agreement with earlier scaling results found in Ref. [8]. In this case, the cosmic-ray source

\footnotetext{
${ }^{1}$ We follow the nomenclature introduced in Ref. [9] and define "spike" to be the steep density enhancement $\rho \sim r^{-A}$ with $A>2$ in the innermost $\sim$ pc due to adiabatic black-hole growth. This should be distinguished from the "cusp," the $\rho \sim r^{-\gamma}$ halo profile found in numerical simulations on scales $\sim$ kpc.
} 
from WIMP pair annihilation, proportional to $\int d^{3} r \rho^{2}(r)$, becomes huge due to the diverging contribution from small radii. (The divergence is cutoff by the black-hole horizon or by a maximum density set by WIMP pair annihilation.) The flux of neutrinos from neutralino annihilation in this spike exceeds current experimental upper bounds in some regions of neutralino parameter space. Subsequent work shows $[12,13]$ that synchrotron emission from the motion of electrons and positrons produced by neutralino annihilation in this spike would also be significant and may exceed current upper bounds. The conclusion of Ref. [12] is that either neutralino dark matter or a cuspy halo must be ruled out. Bertone, Sigl and Silk [13], introducing a different estimate of synchrotron self-absorption, find much weaker, but still significant, limits on the neutralino parameters space. Given these very important implications for dark-matter searches, the claim of the possible presence of a steep dark-matter spike at the galactic center and the underlying assumptions should be investigated more carefully. Here we argue that although a possibility, the enhancement found by GS requires somewhat unusual initial conditions for the galactic halo and for the black hole. In particular, we emphasize how crucial it is that the black hole grew adiabatically from a tiny initial mass to its present-day mass, and that this happened precisely at the center of the dark-matter distribution.

To do so, we consider a few alternatives for the blackhole-formation scenario that we can treat with semi-analytic methods and jet captures the main dynamical processes in (hierarachical) galaxy formation. We show that for the GS spike to arise, the majority of the black-hole growth had to occur within the inner $50 \mathrm{pc}$ of the dark-matter cusp and on a time scale long compared with $10^{7} \mathrm{yr}$. We also show that the growth of the spike relies on the existence of a very high density of cold orbits within the inner $50 \mathrm{pc}$. If the black hole is formed away from the center and then spirals in, it should generically lead to the weaker dark-matter spike, $\rho \propto r^{-0.5}$, with possibly even a depletion, rather than an enhancement, in the central dark-matter density.

The outline of the paper is as follows. In Sec. II, we consider a simple toy model to illustrate the origin of the spike found by GS in case the black hole grows adiabatically at the exact center of the dark-matter system. In Sec. III we perform the computation of the adiabatic growth for a class of more realistic dark-matter-halo profiles, stressing a few more subtle points. In Sec. IV we treat instead the opposite case of fast growth for the black hole. Section V deals with the more complex scenarios of off-centered formation for the black hole. While in all cases above we include just dark matter and the black hole, in Sec. VI we consider the more realistic scenario in which other baryonic components (i.e., stars in the stellar nucleus, bulge, and disk) are included as well. In Sec. VII we summarize and comment on current theoretical ideas about the origin of the black hole.

\section{WARMUP: HALO DISTRIBUTIONS WITH ONLY CIRCULAR ORBITS}

Before proceeding with our numerical calculations, let us consider a simple toy model that illustrates the origin of the
GS spike. In this model, we assume an initial self-gravitating dark-matter distribution $\rho_{i}(r)$ made of particles that are all on circular orbits, at the center of which a black hole grows adiabatically. The angular momentum of each particle remains invariant since the central black hole exerts no torque on any dark-matter particle. This tells us that $r_{i} M_{i}\left(r_{i}\right)$ $=r_{f} M_{f}\left(r_{f}\right)$, where $M(r)$ is the total (i.e., dark-matter plus black-hole) mass enclosed within the radius $r$. Moreover, conservation of the dark-matter mass tells us that $M_{i}^{h}\left(r_{i}\right)$ $=M_{f}^{h}\left(r_{f}\right)$, where the superscript $h$ denotes the halo contribution to the mass.

Now suppose the initial dark-matter density profile is $\rho_{i}\left(r_{i}\right) \propto r_{i}^{-\gamma}$ and let the final density be $\rho_{f}\left(r_{f}\right) \propto r_{f}^{-A}$. Conservation of mass implies that $r_{i}^{3-\gamma} \propto r_{f}^{3-A}$. In the final state, the mass enclosed within a radius $r$ will be dominated by the black hole, $M_{f}\left(r_{f}\right) \simeq M_{\mathrm{BH}}$, as $r \rightarrow 0$. Conservation of angular momentum thus gives $r_{i}^{4-\gamma_{\propto}} r_{f}$. Putting these two conditions together, we find a final profile, $\rho_{f}\left(r_{f}\right) \propto r_{f}^{-A}$, where $A=(9-2 \gamma) /(4-\gamma)[8,9]$. Note that for $0<\gamma<2$, we get $2.25<A<2.5$. Hence the WIMP pair annihilation rate, which is proportional to the density $\rho_{f}\left(r_{f}\right)$ squared, would have diverged except for the truncation at the horizon of the $\mathrm{BH}$.

This derivation illustrates several important points, some of which will be discussed further for more general distributions in subsequent sections.

\section{A. Where does the mass in the spike come from?}

The dark-matter particles that make up the spike come from the inner part of the dark-matter halo, the part that encloses a mass roughly equal to $M_{\mathrm{BH}} \simeq 2.6 \times 10^{6} M_{\odot}$. The addition of a black hole will have negligible effect on circular orbits that enclose larger masses. That the spike comes from this inner part agrees with intuitions, and is suggested by numerical simulation of Ref. [8] (see their Fig. 6) for models with isotropic velocity distributions.

For the power-law density profile that GS considered (and for the Navarro-Frenk-White profile we consider below), the radius that encloses $2.6 \times 10^{6} M_{\odot}$ of halo mass is only about $50-60 \mathrm{pc}$. Thus, for the spike in the GS picture to form, the black hole must have originated within the inner $50-60 \mathrm{pc}$ of the galactic center. This is in some sense surprising because this length scale is microscopic compared with characteristic galactic distance scales: $\sim 3 \mathrm{kpc}$ for the bulge and $\sim 10 \mathrm{kpc}$ for the disk. Put another way, the inner $50 \mathrm{pc}$ encloses only about $(1.5-2) \times 10^{8} M_{\odot}$ of stellar material, about $5 \times 10^{-3}$ of the total stellar mass [probably about $(5-8) \times 10^{10} M_{\odot}$ ] of the galaxy. Although the alignment between the black hole and the stellar distribution is quite good in our own Galaxy (even that is a mystery-the situation is quite different, e.g., in M31), the required alignment is between the black hole and the center of the dark-matter system. Although baryons and dark matter are mixed homogeneously in the early universe, there are numerous processes involved in galaxy formation that should segregate the baryons from the dark matter. For example, baryons will experience radiation drag from the cosmic microwave background when they first start to cool; UV radiation from the first generation of stars could exert a wind on the baryons; in galaxy mergers, baryons 
shock while dark-matter halos pass through each other; baryons today appear in disks, bulges, and bars, unlike dark matter; they are clumped into globular clusters, open clusters, and molecular clouds. Thus, there are many good reasons to believe that the precise center of the baryon distribution should be displaced relative to that of the dark matter, at least at some intermediate stages in the formation of our Galaxy. Thus, even if the black hole formed at the center of a stellar distribution, it would still be somewhat surprising if, at all times during its formation, that coincided to $50 \mathrm{pc}$ with the center of the dark-matter distribution.

\section{B. Cold particles}

The circular-orbit toy model shows us that the particles that give rise to the spike are initially very cold; i.e., the circular speeds in the initial distribution approach zero as $r$ $\rightarrow 0$. (Below, we will show that the same is true for more general and realistic velocity distributions.) Again, this is somewhat surprising, as cold particles are easily subject to heating by a variety of phenomena, especially in the environment at the galactic center. For example, the passing of molecular clouds, open, or globular clusters would tend to disrupt these cold orbits, as would the existence of a bar. Likewise, the mergers and sub-halo accretion that certainly played a role in the formation of the Milky Way would tend to disrupt these cold orbits. We cannot be more quantitative without a more precise model for galaxy formation. However, these disruptive processes must have occurred at some level, and they would tend to hinder the growth of the darkmatter spike.

Note that a dark-matter spike that will give rise to a divergent WIMP annihilation rate can arise even if $\gamma=0$. As explained in Ref. [8], implied above, and discussed further below, the existence of a spike is more generally due to the existence of a singularity in the density of cold orbits at the galactic center. Such singularities can arise even if the density profile is non-singular. If the density $\rho$ at the origin is finite and can be written as an analytic function of the Cartesian coordinates $x, y$, and $z$, then there will be no singularity in the phase-space distribution that gives rise to a spike, but otherwise there will be a phase-space singularity and a spike after adiabatic growth of a black hole [8]. Thus, consider for example, the profiles $\rho(r) \propto(a+r)^{-2}$ and $\rho(r)$ $\propto\left(a^{2}+r^{2}\right)^{-1}$, both of which approach a constant at the origin, and suppose that both have isotropic velocity distributions. As argued in Ref. [8] and described below, adiabatic black-hole growth in the former gives rise to a spike $\rho$ $\propto r^{-A}$ with $A=2$, while the latter gives rise to a spike with $A=1.5$.

\section{Initial black-hole mass}

The circular-orbit model also indicates that the spike will grow only if the black hole grows almost entirely (not just partially) at the black-hole center. Put another way, the spike that grows from an initial state with a $10^{6} M_{\odot}$ black hole and a $\rho \propto r^{-1}$ dark-matter halo to a final state with a $2 \times 10^{6} M_{\odot}$ black hole is not nearly as dramatic as that which arises when a black hole is grown from nothing to $10^{6} M_{\odot}$. If we start with a black hole of significant mass and a dark-matter cusp, then the angular momentum of the inner orbits is dominated by the initial black-hole mass. Slowly doubling the black-hole mass reduces the radius of each of these inner orbits by a factor of 2 and thus leads to a factor-of- 4 density enhancement. In other words, the huge densities of the innermost orbits that give rise to a huge annihilation signal come from the fact that initially, $M_{i}(r) \rightarrow 0$ as $r \rightarrow 0$ and the radius $r_{i}$ of each inner orbit is reduced by a factor $\sim M_{\mathrm{BH}} / M_{i}\left(r_{i}\right)$. If a black hole exists at the galactic center from the start with a mass $M_{\mathrm{BH}}^{i}$ and then grows to a final mass $M_{\mathrm{BH}}^{f}$, the initial dark-matter density profile $\rho_{i}\left(r_{i}\right) \propto r_{i}^{-\gamma}$ becomes a density profile with the same exponent and normalization enhanced by a factor $\sim\left(M_{\mathrm{BH}}^{f} / M_{\mathrm{BH}}^{i}\right)^{3-\gamma}$.

\section{Time scale for adiabatic growth}

If the initial halo profile is $\rho(r) \sim r^{-1}$, then the enclosed mass will be $M(r) \propto r^{2}$, the circular velocity will be $v_{c}(r)$ $\propto r^{1 / 2}$ and the period of a circular orbit will be $T \propto r^{1 / 2}$. The period of an orbit at $50 \mathrm{pc}$ will therefore be $\sim 10^{7} \mathrm{yr}$. Thus, the black hole can grow relatively quickly compared with a cosmological timescale and still be considered adiabatically. This provides an argument in favor of the GS spike. On the other hand, the present-day growth rate of the black hole due to consumption of stars coming inside its loss cone is at most $3 \times 10^{4} M_{\odot} / \operatorname{Gyr}$ [14], one order of magnitude smaller than what is needed to form the current black hole in a Hubble time (mean growth rate of $2 \times 10^{5} \mathrm{M}_{\odot} / \mathrm{Gyr}$ ). This information combined with the evidence that peak active galactic nuclei (AGN) activity occurred near redshift $z \sim 2$ suggests that the majority of the black-hole mass was in place at these early epochs. Although this leaves plenty of time (few Gyr) for the black hole to grow adiabatically, the violent processes that are likely required to form the black holes that power AGN may well operate on a much more rapid time scale.

\section{E. Weaker spikes at smooth densities}

All of the discussion above should make it clear that if the black hole grows adiabatically away from the galactic center, or in a galactic center with a nonsingular phase-space distribution, then the spike should be the weaker $\rho \propto r^{-1.5}$ spike found in Refs. [5-8]. Although this will produce an enhancement in the flux of annihilation radiation, it will not produce the huge enhancement that arises in a spike $\rho(r) \propto r^{-A}$ with $A>2$.

\section{ADIABATIC GROWTH OF THE BLACK HOLE}

We now consider adiabatic growth of a black hole at the center of a spherically symmetric self-gravitating dark-matter system with a more general (and realistic) phase-space distribution. Because of the two key ingredients hereadiabaticity and spherical symmetry-this problem can be treated semi-analytically. The slow changes in the potential allow us to relate in a simple way the dark-matter distribution functions before and after the appearance of the black hole. The symmetry, both in the initial and final states, implies simple forms for the adiabatic invariants. 
The calculation has been discussed in several papers in the past, in the context of growth of a black hole in a general stellar system [5-8], or in the context of growth of the central black hole at the Milky Way center [9] (see also Ref. [7]). Below we briefly review the calculation, both to reproduce the result in GS and to provide some additional insight on the origin of the spike.

\section{A. Semi-analytic treatment}

In a spherically symmetric problem, the adiabatic invariants are the angular momentum and the radial action; i.e., $L_{f}=L_{i} \equiv L$ and $J_{r, f}\left(E_{f}, L\right)=J_{r, i}\left(E_{i}, L\right)$, where [15]

$$
J_{r}(E, L)=\frac{1}{\pi} \int_{r_{\min }}^{r_{\max }} d r v_{r}=\frac{1}{\pi} \int_{r_{\min }}^{r_{\max }} d r \sqrt{2(E-\Phi(r))-\frac{L^{2}}{r^{2}}},
$$

and the subscripts $i$ and $f$ refer to the value of the quantity in the initial and final state. If the growth of the black hole is adiabatic, the distribution function $f(E, L)$ remains unchanged, so $f_{f}\left(E_{f}, L\right)=f_{i}\left(E_{i}, L\right)$.

We specify the initial state by its density $\rho_{i}(r)$ as a function of radius $r$. We also postulate that it has an isotropic velocity-dispersion tensor. If so, then there is a one-to-one correspondence between $\rho_{i}(r)$ and $f_{i}\left(E_{i}\right)$, given by Eddington's formula (see, e.g., [15]),

$$
f(E)=\frac{1}{\sqrt{8} \pi^{2}}\left[\int_{E}^{0} \frac{d^{2} \rho}{d \Phi^{2}} \frac{d \Phi}{\sqrt{\Phi-E}}-\frac{(d \rho / d \Phi)_{\Phi=0}}{\sqrt{-E}}\right],
$$

and the initial state is fully defined.

By inverting the expression for the radial action in the initial state, we can write $E_{i}$ as a function of $E_{f}$ and $L$ and hence find $f_{f}\left(E_{f}, L\right)$ from $f_{i}\left(E_{i}, L\right)$. To compute the radial action in the final state, we actually need first a guess for the final potential. We assume that the potential is dominated by the black hole in its vicinity, while it is roughly unchanged at large galactocentric distances, fixing $\Phi_{f}(r) \cong \Phi_{i}(r)$ $-G M_{\mathrm{BH}} / r$. Once $f_{f}\left(E_{f}, L_{f}\right)$ is obtained, the density profile of the system in the final state is recovered by integrating $f_{f}$ over the velocity space, or, with a change of variables, over $E_{f}$ and $L$ :

$$
\rho_{f}(r)=\frac{4 \pi}{r^{2}} \int_{E_{f}^{\min }}^{0} d E_{f} \int_{L_{f}^{\min }}^{L_{\max }^{\max }} d L_{f} \frac{L_{f}}{v_{r}} f_{f}\left(E_{f}, L_{f}\right)
$$

Because of the form we choose for $\Phi_{f}(r)$, we expect $\rho_{f}(r)$ to be fairly accurate in the central region and far from the black hole. A more accurate result could be obtained in the transition region by iterating. However, we will mainly be interested in the region near the black hole where the approximation $\Phi_{f}(r) \simeq-G M_{\mathrm{BH}} / r$ is fairly accurate. It is also the form adopted by GS.

We apply this procedure numerically in one case. Among the general family of spherical double-power-law darkmatter profiles (the Zhao models [16]),

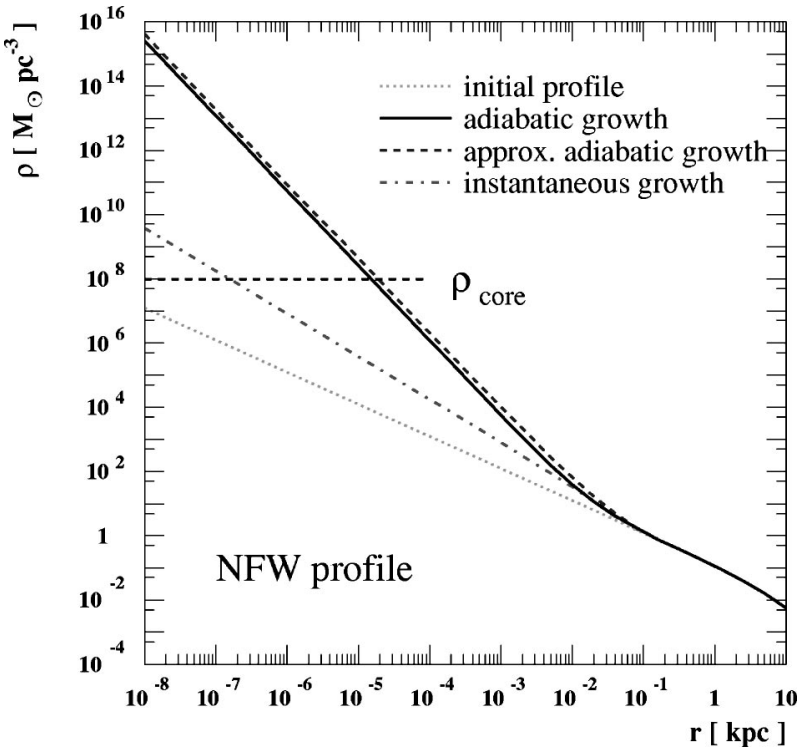

FIG. 1. Enhancement of an NFW dark-matter-density profile due to the growth of the galactic black hole at the center of the dark-matter system. The initial profile (dotted curve) is modified into the solid curve if the growth of the black hole is adiabatic, or into the dash-dot curve for a sudden growth. Also shown is the case of adiabatic growth for an initial profile with all particles on circular orbits (dashed curve). $\rho_{\text {core }}$ is the maximum WIMP density above which WIMPs are depleted by pair annihilations.

$$
\rho_{i}(r)=\rho_{0}\left(\frac{R_{0}}{r}\right)^{\gamma}\left[\frac{1+\left(R_{0} / a\right)^{\alpha}}{1+(r / a)^{\alpha}}\right]^{(\beta-\gamma) / \alpha},
$$

we focus on the functional form suggested by the $\mathrm{N}$-body simulation of Navarro, Frenk, and White (NFW) [10], a density profile with $(\alpha, \beta, \gamma)=(1,3,1)$. We fix the values of the Sun's galactocentric distance, the local halo density and the length scale in the profile, respectively, as $R_{0}=8 \mathrm{kpc}, \rho_{0}$ $=0.3 \mathrm{GeV} \mathrm{cm}^{-3}$ and $a=20 \mathrm{kpc}$ (values compatible with available dynamical constraints); this profile is plotted as a dotted line in Fig. 1.

We first compute numerically the corresponding distribution function and then we follow the same steps applied in Ref. [6] for the isothermal sphere. We perform numerically the inversion between $E_{i}$ and $E_{f}$, computing the radial action for the initial and the final state on grids, respectively, in the $\left(E_{i}, L\right)$ and $\left(E_{f}, L\right)$ plane (we implement adaptive grids in order to achieve everywhere the required numerical accuracy for the inversion). Then we compute $\rho_{f}(r)$ performing the double integral in Eq. (3). The result is shown as a solid line in Fig. 1. As can be seen, the adiabatic growth of the black hole at the center of the dark-matter system induces a sharp increase in the dark-matter density close to the black hole (compare the solid line with the dotted line which gives the initial profile). At a galactocentric distance of about 20 pc, there is the transition from the $1 / r$ cusp in the NFW profile to a much steeper spike $\rho(r) \propto r^{-A}$ with $A=7 / 3$. This is in agreement with the corresponding result (profile scaling as $1 / r^{\gamma}$ with $\left.\gamma=1\right)$ in GS. In Fig. 1 we also plot, as a dashed line, the result for the adiabatic contraction if all particles are 
on circular orbits (the toy model we introduced in the previous section). As can be seen, this simplified treatment reproduces quite closely the result we obtained in case of an isotropic distribution (within a factor of 2, which is less than the uncertainty in the overall normalization of the dark-matter profile).

The density in the spike exceeds $10^{8} M_{\odot} \mathrm{pc}^{-3}$, which is roughly the maximum density if the dark matter is made of WIMPs, which we indicate as $\rho_{\text {core }}$ in this and in the following figures. The limit comes from the value of the pairannihilation cross section for WIMPs, which is fixed, to some extent, by their relic abundance (see GS for details). In fact, the cross section for WIMP-WIMP elastic scattering should generally be larger than the annihilation cross section, so the maximum central density derived by GS should actually be a bit lower.

\section{B. Scaling properties}

The result for the spike slope we have just derived numerically could have been anticipated using scaling properties, as suggested in Ref. [8]. This argument can be summarized as follows. Let the potential of the initial system increase with radius, for small radii, as $\Phi_{i}(r) \sim r^{2-\gamma}$; this is the leading term in systems such as, e.g., those described by Eq. (4) with $\gamma \geqslant 0$. Then the conservation of the radial action implies the scaling $E_{i} \sim E_{f}^{-(2-\gamma) /(4-\gamma)}$ [8]. Suppose then that the distribution function in the initial state diverges near $E_{i}$ $=\Phi_{i}(0)$ as $f_{i}\left(E_{i}\right) \sim\left[E_{i}-\Phi_{i}(0)\right]^{-n}$. Combining this with the expression for the final density profile, Eq. (3), it follows that

$$
\rho_{f}(r) \sim r^{-A}, \quad A=\frac{3}{2}+n\left(\frac{2-\gamma}{4-\gamma}\right) .
$$

It is interesting to derive the exponents $A$ and $n$ for the family of profiles introduced above in Eq. (4). We derive $n$ for $1 \leqslant \alpha \leqslant 2$; results for other ranges can be obtained analogously. In Eddington's formula, Eq. (2), the second term is zero, while the main contribution to the first comes from the singularity in the lower extreme of integration. After some algebra, one finds that the leading terms are given by

$$
f(E) \sim \int_{r^{*}} d r \frac{\left[c_{0}+c_{1}(r / a)^{\alpha}+c_{2}(r / a)^{2 \alpha}+\cdots\right]}{r^{3} \sqrt{r^{2-\gamma}-[E-\Phi(0)]}},
$$

where $r^{*}=[E-\Phi(0)]^{1 /(2-\gamma)}$, and the first two coefficients in the expansion are (up to a constant)

$$
\begin{aligned}
& c_{0}=-2 \gamma(3-\gamma) \\
& c_{1}=(\alpha-2)(3-\gamma)(\beta-\gamma) \frac{(2 \alpha-\gamma)(3-\gamma)+\alpha(\alpha-\gamma)}{\alpha(3+\alpha-\gamma)} .
\end{aligned}
$$

To illustrate, consider three cases. For $\gamma>0$ and independent of $\alpha, c_{0} \neq 0$ and

$$
n=\frac{6-\gamma}{2(2-\gamma)}, \quad A=\frac{9-2 \gamma}{4-\gamma}
$$

recovering the result of Ref. [8] and GS. In the limit $\gamma \rightarrow 0$ and for $1 \leqslant \alpha<2$, the first term in the expansion vanishes, $c_{0}=0$, and hence the second becomes the leading term. In this case one finds milder singularities,

$$
n=\frac{3-\alpha}{2}, \quad A=\frac{9-\alpha}{4} .
$$

Finally, in the limit $\gamma \rightarrow 0$ and $\alpha \rightarrow 2$, both $c_{0}$ and $c_{1}$ go to zero, the distribution function is non-singular, $n=0$, and the density profile after the adiabatic growth of the black hole has a $\gamma=3 / 2$ singularity, which is the result obtained in Ref. [6] using an isothermal (non-singular) distribution function.

A few comments on the results above. The derivation of the scaling in Eq. (5) verifies that the dark-matter spike is mainly formed by particles which, before the black-hole growth, were very cold and close to the galactic center; i.e., particles with $E_{i} \rightarrow \Phi_{i}(0)$. If the distribution function is singular in that limit (i.e., there are many such particles in the initial state), the spike is hugely enhanced. As we have seen, this is possible even for a dark-matter-density profile that is non-singular towards the galactic center (but in this case the spike is always milder than for $\gamma \neq 0$ ). Note also that the enhancement in the spike due to the singularity in the distribution function is possible just if the black hole grows exactly at the center of the initial dark-matter system. If the black hole is formed away from the galactic center, the induced dark-matter spike will be mild. For a system with an isotropic velocity-dispersion tensor, it will be of the same form we obtained above for a non-singular distribution function.

In the previous paragraph, we found for the NFW profile a spike with $A=7 / 3$. For this result to hold, it is crucial to postulate that the initial density profile has a $1 / r$ singularity all the way down to the galactic center. This behavior is just an extrapolation from an $\mathrm{N}$-body simulation with resolution not better than a kpc. As the simulations show that larger structures form from the merging of smaller substructures, such an extrapolation to scales smaller than $10 \mathrm{pc}$ or so may not be trivial. More importantly, even if the $1 / r$ scaling is indeed preserved down to the inner few pc in $N$-body simulations, it is unlikely to survive the addition of baryons. If the density profile is assumed to be truncated in the innermost region, we expect a shallower spike and a model-dependent suppression. For example, if the truncation of a singular profile is performed introducing a core radius, by replacing $\rho_{i}(r) \propto 1 / r^{\gamma}$ with $\rho_{i}(r) \propto 1 /\left[1+(r / a)^{\alpha}\right]^{\gamma / \alpha}$, the spike exponent $A$ will depend just on $\alpha$ (its expression can be recovered from the analysis above substituting $\gamma=0$ and $\beta=\gamma$; note, however, that the normalization of the spike is set by the other parameters as well).

Finally, the results in this section show that the spike obtained by assuming a single-power-law density profile $\left(\sim 1 / r^{\gamma}\right.$ for any $\left.r\right)$ with $\gamma \rightarrow 0$ will probably not provide a good approximation to the true spike. Most likely, the results in Ref. [12], where values of $\gamma$ as low as $10^{-6}$ are considered, will be modified assuming the more general doublepower-law density profile of the form in Eq. (4). 


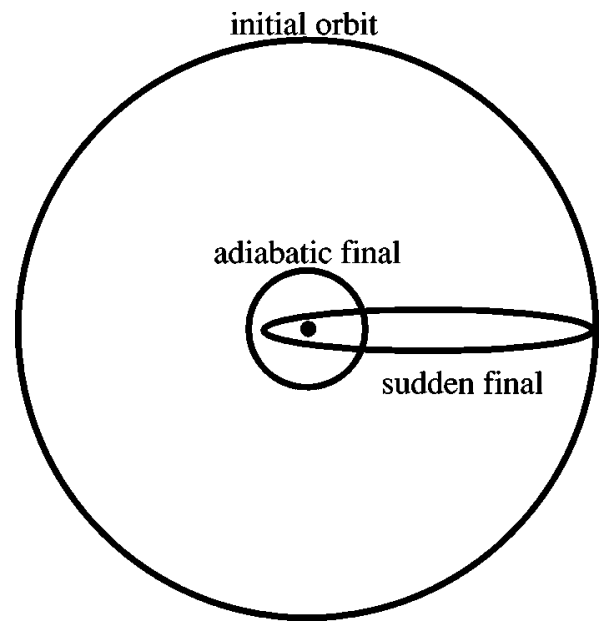

FIG. 2. Initial and final orbits for sudden and adiabatic blackhole growth. If the black-hole grows adiabatically, the initial large circular orbit becomes a final circular orbit of much smaller radius. If the black hole appears suddenly, the initial circular orbit becomes an elliptical orbit shown (assuming that the final potential at these radii is dominated by the black hole).

\section{INSTANTANEOUS GROWTH OF THE BLACK HOLE}

We now consider what happens when the black hole is inserted instantaneously to the center of the dark-matter distribution. This will provide some indication of what happens to the dark-matter distribution if the black-hole growth is too rapid to be approximated as adiabatic. We do this calculation under the assumption that all particles are on circular orbits.

We first provide a qualitative argument to show that the sudden black-hole growth leads to a central dark-matterdensity enhancement that is not as dramatic as that for adiabatic growth. If the black hole grows adiabatically, the initial large circular orbit becomes a final circular orbit of much smaller radius. Thus, the dark matter that was initially at a large radius spends all of its time at a much smaller radius. Now suppose the black hole appears suddenly and dominates the potential at these radii. Again, the angular momentum of the particle is conserved, but its energy changes in such a way that it follows an elliptical Keplerian orbit, as shown in Fig. 2, in which its largest radius is the initial radius. Although the orbit reaches to smaller radii than the circular orbit that arises from adiabatic growth, the velocity of the particle at these small radii is large. As a result, the particle on this orbit spends most of its time at radii larger than the radius of the final orbit from adiabatic growth.

We now quantify these arguments. When the black hole is added, the velocity of each particle remains unchanged, but the gravitational potential is changed suddenly. The final profile is obtained by following each particle along its subsequent orbit. The probability for a particle that at the appearance of the black hole was at galactocentric distance $r_{0}$ with a velocity $\vec{v}_{0}$ to be between radii $r$ and $r+d r$ is

$$
P(r) d r=\frac{d t}{T\left(r_{0}, \vec{v}_{0}\right)}=\frac{1}{T\left(r_{0}, \vec{v}_{0}\right)} \frac{d r}{v_{r}\left(r, r_{0}, \vec{v}_{0}\right)},
$$

where $T$ is half of the period of the particle, and $v_{r}$ is the radial component of its velocity.

If all particles are on circular orbits, then the calculation is simplified, as the initial radial velocities are all zero. We thus need to integrate the probability that a particle that is observed at radius $r$ in the final configuration came from an orbit at radius $r_{0}$ in the initial configuration. Doing so, we find that the final radial profile is given by

$$
\rho_{f}(r)=\frac{1}{r^{2}} \int_{r_{\min }}^{r_{\max }} d r_{0} r_{0}^{2} \rho_{i}\left(r_{0}\right) \frac{1}{T\left(r_{0}\right)} \frac{1}{v_{r}\left(r, r_{0}\right)},
$$

where

$$
v_{r}=\sqrt{2\left[\Phi_{f}\left(r_{0}\right)-\Phi_{f}(r)\right]+v_{c}^{2}\left(r_{0}\right)\left(1-r_{0}^{2} / r^{2}\right)},
$$

with $v_{c}$ the circular velocity in the initial state. The extremes of integration $r_{\min }$ and $r_{\max }$ are the minimum and maximum initial radii for particles contributing to the final profile at the radius $r$. For such radii, $v_{r}\left(r, r_{\min }\right)=v_{r}\left(r, r_{\max }\right)=0$. From the expression for $v_{r}$, it follows that $r_{\min }=r$; i.e., a particle on a circular orbit shrinks in general to a rosette orbit with apocenter equal to the radius of the initial orbit.

We compute $\rho_{f}$ for the initial NFW profile introduced in Sec. III A. Again, we assume $\Phi_{f}(r) \cong \Phi_{f}(r)-G M_{\mathrm{BH}} / r$; the result is shown as a dash-dot curve in Fig. 1. Although the black hole steepens the profile, the steepening is not nearly as dramatic as if the black hole is introduced adiabatically.

The final profile has a $r^{-4 / 3}$ singularity, a result that can be understood analytically. In the small- $r$ limit, the potential is dominated by the black hole, and $T\left(r_{0}\right) \propto r_{0}^{3 / 2}$, while $v_{r}$ becomes

$$
v_{r}=\sqrt{\frac{2 G M_{\mathrm{BH}}}{r r_{0}}} \sqrt{\left(r_{0}-r\right)\left(1-\frac{M_{i}^{h}\left(r_{0}\right)}{2 M_{\mathrm{BH}}}\left(1+\frac{r_{0}}{r}\right)\right)} .
$$

For the NFW profile, the halo mass $M_{i}^{h}\left(r_{0}\right)$ enclosed within the radius $r_{0}$ is proportional to $r_{0}^{2}, r_{\max }$ can be computed exactly, and the final profile is

$$
\begin{aligned}
\rho_{f}(r) \sim & \frac{1}{r} \int_{r}^{r_{\max }} d r_{0} \frac{1}{\sqrt{\left(r_{0}-r\right)\left(r_{\max }-r_{0}\right)}} \\
& \times \frac{1}{\sqrt{r_{0}^{2}-\left(r r_{0}\right) /\left(k r_{\max }^{2}\right)-r /\left(k r_{\max }\right)}} \\
& \sim \frac{1}{r^{4 / 3}},
\end{aligned}
$$

as found numerically and shown in Fig. 1.

Heuristically, our circular-orbit model should generalize to any orbit with a finite peri-to-apocenter ratio. The apocenter distribution is unchanged after the implanting of the black hole, and the pericenter is determined by the angularmomentum distribution, which is a power law differing from that of the circular orbits by a scaling. So we expect the 


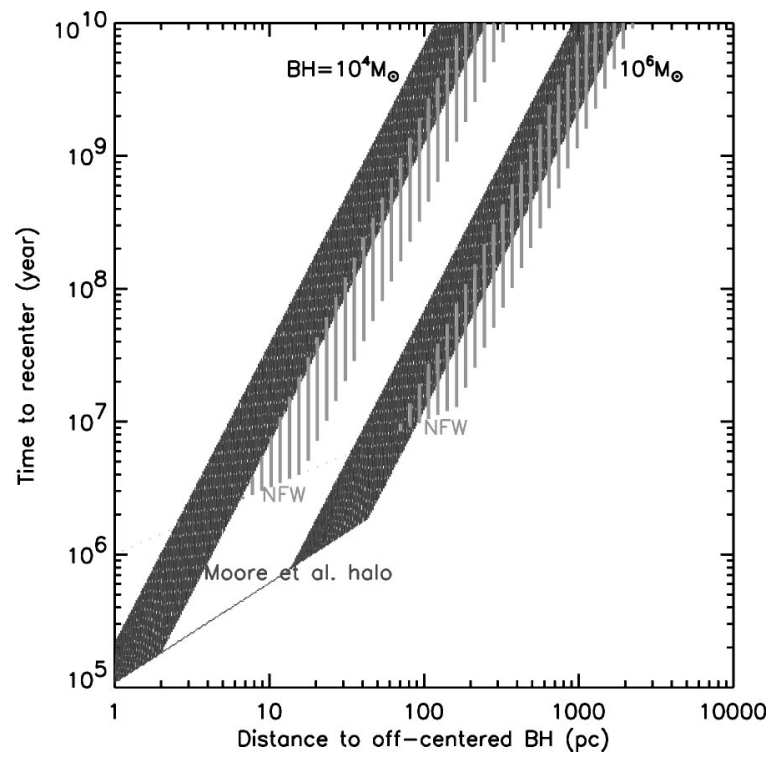

FIG. 3. Predicted time for a seed black hole of mass $M_{\mathrm{BH}}^{S}$ $=10^{4} M_{\odot}$ and $10^{6} M_{\odot}$ to spiral into the galactic center from a radius $r$ in an initially pure NFW or pure Moore halo profile (no baryons). The shaded regions show the range estimated with Chandrasekhar's formula for dynamical friction, which is slow for a naked $\mathrm{BH}$ and can be speeded up by a factor of as much as 6 if the BH comes with its own mini-halo (Milosavljevic and Merritt 2001). Note it takes longer than a Hubble time for a $10^{4} M_{\odot}$ black hole to spiral from $r \gg 100 \mathrm{pc}$, and for a $10^{6} M_{\odot}$ black hole to spiral from $r \gg 1 \mathrm{kpc}$. At very small radius when the enclosed halo mass becomes comparable to the mass of the infalling hole, the hole would fall in quickly on a dynamical time scale, which is independent of $M_{\mathrm{BH}}^{S}$.

pericenter to move in according to the same power law as for circular orbits and thus produce a density power law as before except for normalization.

\section{MODELS WITH OFF-CENTERED BLACK HOLE}

The black hole, or at least its seed, might not form exactly at the center of the dark-matter halo due to deviations from spherical symmetry in various galaxy formation processes. If the black hole forms off-center, then it will slowly sink via dynamical friction to the center of the galactic potential well. We sketch in this section the number of competing effects entering this problem.

\section{A. Enough time to spiral in?}

The process of dynamical friction is not efficient enough to center the black hole regardless of its initial mass and of its initial distance from the center of mass. The spiral-in time scale for a very small black hole or for a black hole born very far from the center of the Galaxy, may be longer than the age of the Galaxy itself. This is illustrated in Fig. 3 where we show the spiral-in time scale for black holes that are initially isolated and initially surrounded by stellar cusps. Naively, if the black hole is initially surrounded by a stellar cusp, then the spiral-in time scale should be about one half of the time scale in case of an isolated black hole; more precisely, according to recent numerical simulations, the time scale is reduced by a factor of 6 [17] (with the extra factorof-3 reduction still not understood). For a black-hole seed of mass $10^{4} M_{\odot}$, the time scale to spiral in the potential well of the NFW profile we considered is roughly comparable to the Hubble time if its initial location is $200 \mathrm{pc}$ away from the center of the dark-matter system. Also shown is the case of a black-hole seed of mass $10^{6} M_{\odot}$, for which the timescale is of the order of $10 \mathrm{Gyr}$ if it spirals in from $1 \mathrm{kpc}$. The conclusion is that if, as plausible, the black-hole seed is generated through some violent process off-center, it will not get to the center until almost fully formed.

\section{B. Merger and growth at the center}

What happens when the seed $\mathrm{BH}$ arrives at the center? There are two competing effects with the dark-matter distribution near the galactic center: (a) it may be smoothed out by the time-dependent perturbation induced by the incoming black-hole; (b) it may be squeezed in by subsequent at-thecenter growth of the black hole.

Effect (a) has been investigated with $N$-body simulations in Ref. [18]. It was shown there that the back reaction to the spiral-in of the black hole leads to the formation of a weak density cusp, with an $r^{-0.5}$ singularity, regardless of the initial density profile. More precisely, the same features are obtained in simulations with the King model (which has a core radius) and with the Hernquist profile [which is singular at the galactic center and given by Eq. (4) with $(\alpha, \beta, \gamma)$ $=(1,4,1)]$. Furthermore, the size of the weak-cusp region is found to be correlated to the black-hole mass, with roughly the total mass of the region affected by the black hole equal to the black-hole mass. The proposed explanation for this result is as follows: The sinking of the black hole induces a heating process that leaves no particle in the system with energy below some minimum energy $E_{0}$. We saw above in the case of adiabatic growth at the center of the halo that the spike in the final density profile was due to initially cold particles. If such particles are removed from the system, neglecting the dependence on angular momentum in the distribution function, one finds that the final density profile goes at small radii like [18]

$$
\begin{aligned}
\rho_{f}(r) & =4 \pi \int_{\Phi_{f}(r)}^{0} f_{f}\left(E_{f}\right) \sqrt{2\left[E_{f}-\Phi_{f}(r)\right]} \\
& =4 \pi \int_{E_{0}}^{0} f_{f}\left(E_{f}\right) \sqrt{2\left[E_{f}-\Phi_{f}(r)\right]} \\
& \sim \sqrt{-\Phi_{f}(r)} \sim \sqrt{\frac{1}{r}} .
\end{aligned}
$$

We implement this result in our picture, with a simple scheme. We suppose we have an initial dark-halo profile of the form in Eq. (4). Then we postulate the off-center formation of a black-hole seed of mass $M_{\mathrm{BH}}^{S}$, which we keep as a free parameter. Since we are supposing that the black-hole seed appears off-center, regardless of whether it forms quickly or slowly, it induces a very mild dark-matter spike around it; we neglect this effect. We model then the modifi- 


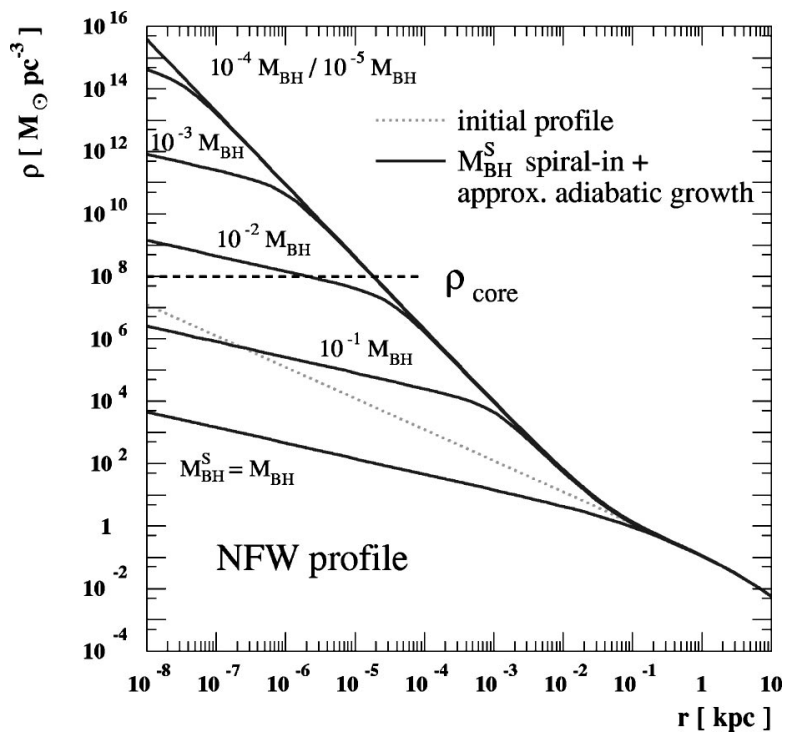

FIG. 4. Modification of an NFW dark-matter-density profile due to the off-center formation of a black-hole seed of mass $M_{\mathrm{BH}}^{S}$, its spiral in the center of the dark-matter system and its adiabatic growth to the present-day mass of the black hole at the galactic center. The cases for a few different values for the black-hole-seed mass are plotted. $\rho_{\text {core }}$ is the maximum WIMP density above which WIMPs are depleted by pair annihilations.

cation to the dark-halo profile due to the sinking of the blackhole seed in the center of the Galaxy by replacing the initial density profile with the following:

$\rho_{\text {int }}(r)=\rho_{0}\left(\frac{R_{0}}{r}\right)^{0.5}\left[\frac{1+\left(R_{0} / r_{s}\right)}{1+\left(r / r_{s}\right)}\right]^{\gamma-0.5}\left[\frac{1+\left(R_{0} / a\right)^{\alpha}}{1+(r / a)^{\alpha}}\right]^{(\beta-\gamma) / \alpha}$,

which is essentially a modified Zhao model with a break at both $r_{s}$ and $a$. The new length scale $r_{s}$ we introduce is determined by requiring that the mass of the dark halo within $r_{s}$ to be equal to $M_{\mathrm{BH}}^{S}$.

Moving onto effect (b), we model the accretion of matter onto the black hole so that $M_{\mathrm{BH}}^{S}$ grows to the mass we now observe $M_{\mathrm{BH}}$ as a slow adiabatic process, and compute the final dark-matter density, treating the adiabatic contraction in the circular-orbit approximation described above.

In Fig. 4 we plot $\rho_{f}$ for the initial NFW profile introduced in Sec. III A and for a few choices for the mass of the blackhole seed. As can be seen, if the black hole that spirals in has a mass comparable to (more than a tenth of) the mass we observe now at the center of the Galaxy, the dark-matter density close to the black hole is, in this picture, lower than the density in the initial profile. This indicates that the number of dark-matter particles expelled from the inner Galaxy by the black-hole seed is larger than the number of those attracted later on by the adiabatic deepening of the potential well due to the increase in mass of the black hole.

The adiabatic contraction process dominates for $M_{\mathrm{BH}}^{S}$ $\lesssim M_{\mathrm{BH}} / 10$. For $M_{\mathrm{BH}}^{S} \lesssim M_{\mathrm{BH}} / 200$, the end result is a dramatic enhancement in the dark-matter density around the black hole. Although the final density profile obtained with this

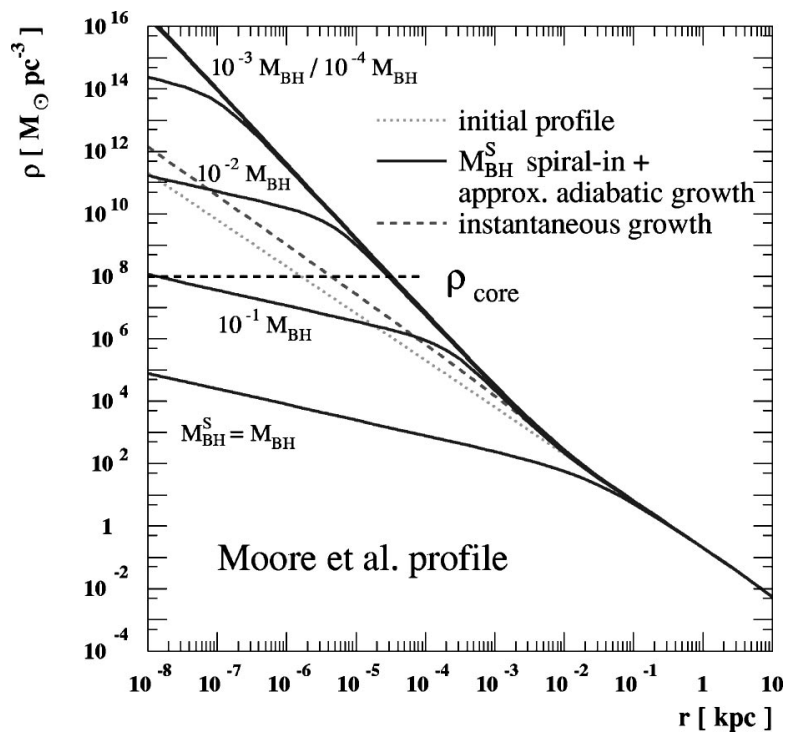

FIG. 5. The same as in Fig. 4, but for a Moore et al. darkmatter-density profile. Also shown is the modification of the profile for sudden growth of the black hole at the center of the dark-matter system (dashed curve).

procedure differs from what we found in case of adiabatic growth of the black hole at the center of the dark-matter system, the difference occurs only at densities higher than the maximum WIMP density $\rho_{\text {core }}$. Thus, if a black-hole seed with less than 1/200th the mass of the final black hole is let to grow adiabatically at the center, then a spike that is essentially indistinguishable accordance with the GS result could be formed. The trouble is that such small seed cannot spiral in the center quickly enough, as shown by Fig. 3. So the conclusion is that if the GS spike is to grow, the black hole must form within the inner $50 \mathrm{pc}$ of the dark-matter distribution: If it forms outside this radius with a large mass, it will destroy the cusp as it spirals in, and if it forms well outside this radius with a small mass, it will not have enough time to spiral in.

Although we have considered so far just the NFW profile, the procedures we outlined can be applied to any density profile. As another example, we consider the dark-matterdensity profile obtained in the high-resolution $\mathrm{N}$-body simulation of Moore et al. [11]. In this case, the initial darkmatter profile is more cuspy towards the galactic center and is given by Eq. (4) with $(\alpha, \beta, \gamma)=(1.5,3,1.5)$ (hereafter the Moore et al. profile). Again we make a choice for the values of our galactocentric distance, the local halo density, and the length scale in agreement with available dynamical constraints: $R_{0}=8 \mathrm{kpc}, \rho_{0}=0.3 \mathrm{GeV} \mathrm{cm}^{-3}$, and $a=28 \mathrm{kpc}$.

Results in this case are shown in Fig. 5. The initial profile is shown as a dotted curve; the dashed curve shows the slight increase in density in case of instantaneous growth of the black hole according to the treatment in Sec. IV. The solid curves give the final profile in the case described above in this section. We see again that for large values of the mass of the black-hole seed, the net effect is a decrease in the darkmatter density. For values $M_{\mathrm{BH}}^{S} \lesssim M_{\mathrm{BH}} / 50$, the enhancement in the final density reproduces, for $\rho_{f}<\rho_{\text {core }}$, the result one 
gets just assuming the adiabatic growth at the center of the dark matter system. The time scales to re-center black hole seeds of $10^{4} M_{\odot}$ and $10^{6} M_{\odot}$ are again shown in Fig. 3, and the same discussion we outlined for the NFW case applies here.

\section{TAKING STARS INTO ACCOUNT}

So far we have used a schematic picture of the Galaxy which included just dark matter and the black hole, neglecting other baryonic components. More realistically, the stellar components may outweigh the dark matter in the inner part of the Galaxy in the initial configuration before the black hole grows. We treat this problem here.

In the standard sequence of galaxy formation, a roughly spherical overdensity, in which the baryons and dark matter are distributed homogeneously, undergoes gravitational collapse. The collisionless matter then relaxes to an NFW or Moore profile, as indicated by $N$-body simulations, while the baryonic matter shocks, heats, and then cools to a disk and/or bulge in the gravitational potential well generated by both the dark and the baryonic matter. The radiative cooling and collapse of baryons is likely to be a slow process and hence it should be a good approximation to treat it as an adiabatic contraction of the baryons.

We sketch this process in one example. We reconsider again the case of off-center formation discussed in the previous section, refining the definition of initial and final states. In the initial state we add the extra component due to baryons (same density profile as for dark matter but scaled down of a factor $\Omega_{b} / \Omega_{M}$ ). In the final state we add the stellar bulge and disk. We pick standard forms for them, compatible with available observations in the outer part of the Galaxy and with the rotation curve close to the black hole (we do not reproduce the functional forms here as they do not enter critically in the discussion below). The spiral-in of the blackhole seed is treated in the same way as in Sec. V. The adiabatic contraction is performed using the circular-orbit approximation. We also replace the disk with a spherical mass distribution that produces the same rotation curve. This simplification allows us to still treat the problem as spherically symmetric and does not enter crucially in the results we focus on.

Results are shown in Fig. 6. The adiabatic contraction of the baryons in the bulge and the disk induces a significant enhancement of the dark-matter density at intermediate galactocentric distances (few pc up to few $\mathrm{kpc}$ ). In the innermost region, however, the main role is still played by the black hole.

\section{CONCLUSIONS}

We have tried to determine the initial conditions required for growth of this spike by considering several alternative formation scenarios. We have found that for the spike to arise, the majority of the black-hole mass must grow on a time scale long compared with $10^{7} \mathrm{yr}$ and within $50 \mathrm{pc}$ of the center of a cuspy dark-matter distribution. Moreover, the dark-matter particles that make up this spike must be initially

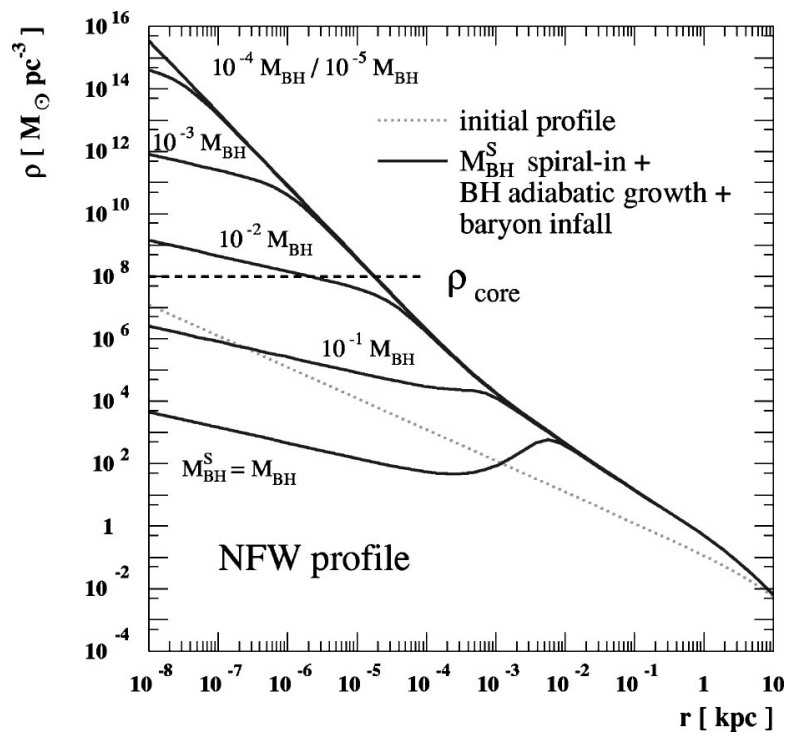

FIG. 6. The same as in Fig. 4, but taking into account the adiabatic contraction of baryons that form the bulge and the disk.

in very cold orbits near the galactic center. A spike essentially equivalent to the GS spike could in principle arise if a low-mass black hole spiraled in to the galactic center and then grew in mass, but the spiral-in time scale for such lowmass black holes is larger than the Hubble time.

If we understood precisely how the black hole in the galactic center formed, we could say more definitively whether the GS spike arises or not. Unfortunately, however, the details of black-hole formation are far from clear. The presentday growth rate for the Milky Way's black hole as well as the peak of AGN activity at redshifts $z \sim 2$ suggest that a good fraction of the black-hole mass was in place at early times. The most promising mechanisms for black-hole production at high redshift do not seem to contain the ingredients required for the GS spike (see, e.g., Ref. [19]). $N$-body simulations show that at that time, dark halos are forming via rapid and often violent mergers. Moreover, hydrodynamic simulations show that when two galaxies collide, they can leave behind a cold dense baryonic gas that can then collapse to form a supermassive black hole. The rate for black-hole growth is estimated to be orders of magnitude larger than the current rate (see, e.g., Ref. [20]), although a time scale long compared with $10^{7} \mathrm{yr}$, the period for the orbits that eventually wind up in the spike, is still conceivable. However, even if the time scale is reasonable, the dark halos for each of the two initial galaxies pass through each other almost undisturbed and then later relax to the dark-matter profile for a single halo. The timescale for this relaxation should be even longer.

Moreover, it is hard to believe that the cold orbits required for the GS spike would be found right at the center of the dark-matter distribution so soon after this very violent collision. The mass density in these inner regions will be dominated by the baryons, and baryons can shock, form molecular clouds, stars, star clusters, and supernova-driven winds. The dynamical effects of all these processes are heuristically expected to disrupt cold orbits. Also, it is not clear why the 
centers of the dark-matter and gas distributions should be so coincident in this violent formation. (Although the black hole in our Galaxy coincides fairly well at the current epoch with what appears to be the dynamic center of the Galaxy, this is not necessarily the case in other galaxies, such as M31.)

An alternative (although not mutually exclusive) possibility is that a $10^{3}-10^{5} M_{\odot}$ black hole could form at a redshift $z \sim 30$ when molecular-hydrogen cooling permits the collapse of the first baryonic objects. The remaining $10^{6} M_{\odot}$ of mass could then be accreted during the subsequent generations of mergers that eventually give rise to a galactic halo. Although it would not be unusual for the initial high-density peak in the primordial mass distribution that gave rise to this black-hole seed to be near the center of the resulting galactic halo, it would be somewhat surprising if it was so close to the center.

Finally, another possibility is that the black hole at the galactic center was formed from the merger of two smaller black holes that would have followed the merger of two cuspy halos, each with its own black hole. Simulations by Barnes [21] show that if two cuspy halos form, then the cusp may be preserved in the merged halo. However, recent highresolution simulations by Milosavljevic and Merritt [17] show that when black holes are included, the formation of a hard black-hole binary knocks particles out of the inner cusp and reduces the initial power-law profile to a shallower profile. This is precisely the disruption of cold orbits to which we were referring above. Although these simulations deal with cusps, and not spikes, they do seem indicate that the innermost orbits are disturbed and the innermost density decreased during the final stages of a merger.

In conclusion, we have determined the initial conditions for the growth of the GS spike and given several reasons to doubt they were manifest in our own Galaxy. Given these doubts, null searches for annihilation radiation from the galactic center should not be interpreted as evidence against WIMP dark matter, nor against a cuspy halo.

\section{ACKNOWLEDGMENTS}

We thank A. Loeb for useful discussions. This work was supported in part at Caltech by NSF AST-0096023, NASA NAG5-8506, and DOE DE-FG03-92-ER40701, and at Trieste by the RTN project under grant HPRN-CT-2000-00152. H.S.Z. wishes to thank Institut d'Astrophysique de Paris and Sterrewacht Leiden for financial support and Caltech for hospitality during his visit.
[1] G. Jungman, M. Kamionkowski, and K. Griest, Phys. Rep. 267, 195 (1996).

[2] L. Bergstrom, Rep. Prog. Phys. 63, 793 (2000).

[3] J. Kormendy and L. C. Ho, astro-ph/0003268.

[4] A. Eckhart and R. Genzel, Nature (London) 383, 415 (1996); B. M. Klein et al., Astrophys. J. 509, 678 (1998); R. Genzel et al., astro-ph/001428.

[5] P. J. E. Peebles, Gen. Relativ. Gravit. 3, 63 (1972).

[6] P. Young, Astrophys. J. 242, 1232 (1980).

[7] J. R. Ipser and P. Sikivie, Phys. Rev. D 35, 3695 (1987).

[8] G. D. Quinlan, L. Hernquist, and S. Sigurdsson, Astrophys. J. 440, 554 (1995).

[9] P. Gondolo and J. Silk, Phys. Rev. Lett. 83, 1719 (1999).

[10] J. F. Navarro, C. S. Frenk, and S. D. M. White, Astrophys. J. 462, 563 (1996).
[11] B. Moore et al., Astrophys. J. Lett. 499, L5 (1998).

[12] P. Gondolo, Phys. Lett. B 494, 181 (2000).

[13] G. Bertone, G. Sigl, and J. Silk, astro-ph/0011553; astro-ph/0101134.

[14] J. Magorrian and S. Tremaine, Mon. Not. R. Astron. Soc. 309, 447 (1999).

[15] J. Binney and S. Tremaine, Galactic Dynamics (Princeton University Press, Princeton, NJ, 1987).

[16] H. S. Zhao, Mon. Not. R. Astron. Soc. 278, 488 (1996).

[17] M. Milosavljevic and D. Merritt, astro-ph/0103350.

[18] T. Nakano and J. Makino, Astrophys. J. Lett. 525, L77 (1999).

[19] R. D. Blandford, astro-ph/9906025; M. J. Rees, astro-ph/9912346.

[20] H. S. Zhao et al. (in preparation).

[21] J. Barnes, astro-ph/9811091. 